\title{
Study on contact angle of double-decker bearing with consider of centrifugal expansion
}

\author{
Jing Hu, XiaoLi Qiao, QiongYing Lv, XinMing Zhang*, JiuTong Zhai, HaiLong Li \\ Ministry of Education Key Laboratory for Cross-Scale Micro and Nano Manufacturing, Changchun University of Science and \\ Technology, China.
}

\begin{abstract}
Improving the precision of numerical calculation of double-decker bearing is the basis of exploring its potential application value. This paper takes double-decker bearing as the research object, a modified quasi-statics model of double-decker bearing was established by considering the influence of the centrifugal expansion of the inner and middle ring of double-decker bearing at high speed and solve it with soft of Matlab. The influence of centrifugal expansion on the contact angle of the double-decker bearing was analyzed. The results show that the centrifugal expansion caused by the inner ring and middle ring under high speed can not be ignored, and the centrifugal expansion has a greater influence on the contact angle of inner bearing than that of outer bearing. The influence of centrifugal expansion on the dynamic properties of different materials is different, and the change of contact angle caused by ceramic as the inner ring and middle ring is greater than that of steel. It provides the basis for more accurate numerical analysis and design of the double-decker bearing.
\end{abstract}

\section{Introduction}

Rolling bearings are the core parts of the reliable operation of mechanical equipment. To further improve the performance of bearings, explore the potential application value of bearings, Har Prashad proposed the concept of double-decker rolling bearings in 2001[1]. Since its introduction, some scholars have done some research on numerical analysis. In 2004, Har Prashad regarded the double-decker bearing as a single-layer rolling bearing with some equivalent pitch diameter and equivalent ball diameter [2]. In 2014, Chengtao Yu analyzed the mechanical properties of the "Z" type of double-decker ball bearing based on Hertz basic theory and safe contact angle theory [3]. In 2016, Yili Zhu proposed a new type of "I" bearing in the prototype of double-decker bearing and themechanical model is established [4]. With the increasing demand for the strength, reliability and precision of the double-decker bearing, it is of great significance to predict its dynamic performance more accurately and practically.

Centrifugal expansion is a phenomenon that with the increase of rotating speed and the action of centrifugal force, the stress inside the parts will be redistributed and elastic expansion deformation occurs. Centrifugal expansion can change the contact state of the bearing, which affects the dynamic performance of the bearing. In recent years, many scholars have paid attention to the factors of centrifugal expansion and conducted relevant researches. In 2004, Hongqi Li proposed an integrated

\footnotetext{
*Corresponding author: zxm@cust.edu.cn
}

thermodynamic model applicable to various high-speed spindles and considered the influencing factors of centrifugal expansion rate [5]. In 2012, Hongrui Cao studied the effect of centrifugal expansion on the dynamic performance of single-layer bearings [6]. In 2014, Aleksandar Zivkovic established a thermalmechanical model of the angular contact ball bearing spindle, in which the quasi-static model considered the centrifugal expansion rate, and discussed the influence of the predicted temperature distribution of the spindle on its static characteristics and the influence of the thermal expansion of the spindle on the machining accuracy[7]. Compared with single-layer bearing, the numerical analysis of double-decker bearing is less, and the factor of centrifugal expansion is not considered. The improvement of the precision of the numerical analysis model is of great significance to the study of its potential application value.

In this paper, considering the factor of centrifugal expansion, a modified quasi-static model of doubledecker bearing is established. The influence of the contact angle on the double-decker bearing model is shown here.

\section{Analysis of centrifugal expansion}

The width of the inner ring of the ball bearing is usually much smaller than the diameter. The inner ring of the bearing can be regarded as a hollow ring. Meanwhile, it is assumed that the temperature of the ring is ax symmetric and evenly distributed in an axial direction. In 
the cylindrical coordinate system, the equilibrium relation of the ring is as follows:

$$
\frac{d \sigma_{a}}{d R}+\frac{\sigma_{a}-\sigma_{b}}{R}+\rho \omega^{2} R=0
$$

Where, $\sigma_{a}$ is the radial stress component, $\sigma_{b}$ is the tangential stress component, $R$ is the radius of bearing inner ring, $\omega$ is the rate of angular motion; $\rho$ is the density of inner ring material.

The constitutive equation of elasticity and the geometric relation is:

$\varepsilon_{a}=\frac{\sigma_{a}-v \sigma_{b}}{E}, \varepsilon_{b}=\frac{\sigma_{b}-v \sigma_{a}}{E} \quad \varepsilon_{a}=\frac{d \varsigma}{d R}, \varepsilon_{b}=\frac{\varsigma}{R}$

Where, $\varsigma$ is the elastic deformation.

Represents the coordination relation of the stress component as follows:

$$
\sigma_{b}-\sigma_{a}+R \frac{d \sigma_{b}}{d R}=0
$$

At high speed, the radial expansion displacement of the bearing inner ring is[7][8]:

$$
\psi_{R}=\rho \omega^{2} d_{m}\left[D_{i}^{2}(3+v)+d_{m}^{2}(1-v)\right]
$$

Where, $d_{m}$ is the pitch diameter, $D_{i}$ is the inner diameter, $v$ is the Poisson ratio.

\section{Quasi-static m odel of double-decker bearing and its modification}

\subsection{Quasi-static model}

The double-decker ball bearing is composed of two layers of the ball bearing. Its relevant geometric parameters are shown in Fig. 1. The geometric relationship is shown in formula (5)(12)[9].

\subsubsection{Deformation coordinate condition}

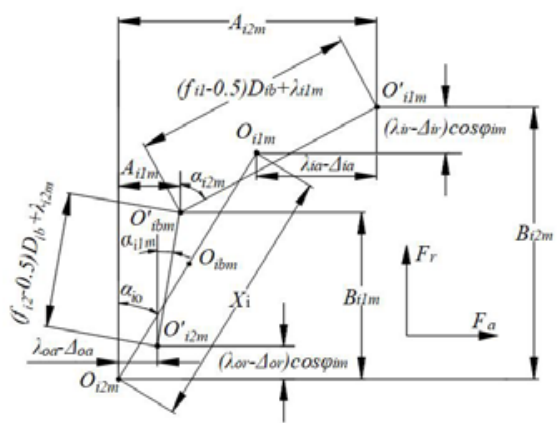

(a)The Deformation coordinate condition of inner ball bearing

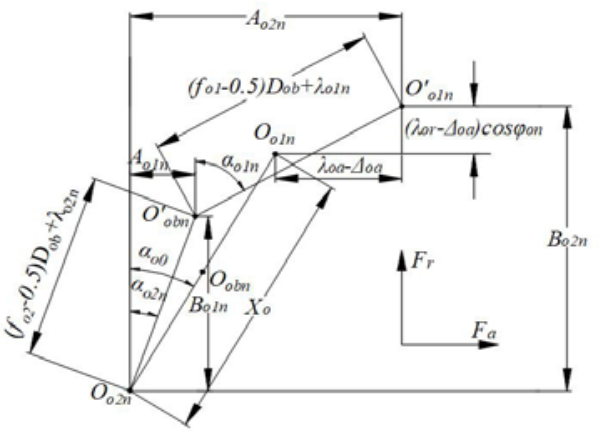

(b)The Deformation coordinate condition of outer ball bearing

Fig.1. The position relation diagram of ball center and curvature center of the raceway in double-decker bearing

$$
\begin{gathered}
\lambda_{i 2 m}=\sqrt{A_{i 1 m}^{2}+B_{i 1 m}^{2}}-\left(f_{i 2}-0.5\right) D_{i b} \\
B_{i 1 m}=X_{i} \cos \alpha_{i 0}+\left(\lambda_{o r}-\Delta_{o r}\right) \cos \varphi_{i m} \\
\lambda_{i 2 m}=\sqrt{A_{i 1 m}^{2}+B_{i 1 m}^{2}}-\left(f_{i 2}-0.5\right) D_{i b} \\
\lambda_{i 1 m}=\sqrt{\left(A_{i 2 m}-A_{i 1 m}\right)^{2}+\left(B_{i 2 m}-B_{i 1 m}\right)^{2}}-\left(f_{i 1}-0.5\right) D_{i b}
\end{gathered}
$$

$$
\begin{gathered}
A_{o 2 n}=X_{o} \sin \alpha_{o 0}+\lambda_{o a}-\Delta_{o a} \\
B_{o 1 n}=X_{o} \cos \alpha_{o 0}+\left(\lambda_{o r}-\Delta_{o r}\right) \cos \varphi_{o n} \\
\lambda_{o 2 n}=\sqrt{A_{o 1 n}^{2}+B_{o 1 n}^{2}}-\left(f_{o 2}-0.5\right) D_{o b} \\
\lambda_{o 1 n}=\sqrt{\left(A_{o 2 n}-A_{o 1 n}\right)^{2}+\left(B_{o 2 n}-B_{o 1 n}\right)^{2}}-\left(f_{o 1}-0.5\right) D_{o b}
\end{gathered}
$$

Where, $\alpha_{i 0}, \alpha_{o 0}$ is the initial contact angle; $\varphi_{i m}, \varphi_{o n}$ is the ball position angle; $\Delta_{o r}, \Delta_{o a}$ is the radial and the axial clearance; $\lambda_{o 1 n}, \lambda_{o 2 n}, \lambda_{i 1 m}, \lambda_{i 2 m}$ is the contact deformation; $\lambda_{\text {ia }}, \lambda_{\text {oa }}, \lambda_{\text {ir }}, \lambda_{\text {or }}$ is the axial and the radial displacement; $X_{i}, X_{o}$ is the distance between the centre of curvature and the raceway; $B_{i 1 m}, B_{i 2 m}$ is the radial projection; $A_{i 1 m}, A_{i 2 m}$ is the axial projection; $A_{o 1 n}, B_{o 1 n}$ is the axial and radial projections between the curvature centre of the inner and outer rings of double-decker bearing inner bearing after loading; $\alpha_{i 1 m}, \alpha_{o 1 n}, \alpha_{i 2 m}, \alpha_{o 2 n}$ is the inner contact angle.

\subsubsection{Force equilibrium equation}

The force diagram of the double-decker bearing is shown in Fig. 2. The analysis model is similar to that of the single-layer bearing. The difference lies in the mid-ring equilibrium formula and the speed distribution between the inner ring and the mid-ring. The relation between inner and middle rotation speeds is shown in formula (13). Assuming that the bearing is affected by axial and radial forces. The force balance equation of the middle ring is shown in formula (14)-(15).

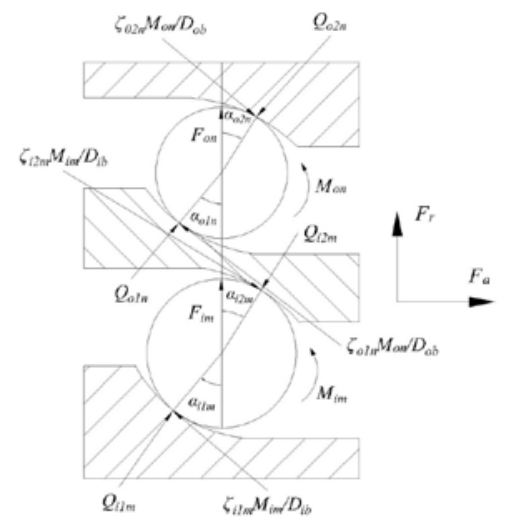


Fig. 2. The force diagram of double-decker bearing

$$
\begin{gathered}
n_{m}=\frac{D_{i p}{ }^{4.5}}{D_{i p}{ }^{4.5}+D_{o p}{ }^{4.5}} n_{i} \\
\sum_{n=1}^{Z_{o b}}\left[Q_{o 1 n} \cos \alpha_{o 1 n}+\frac{\xi_{o 1 n} M_{o n}}{D_{o b}} \sin \alpha_{o 1 n}\right] \cos \varphi_{o n}= \\
\sum_{m=1}^{Z_{i b}}\left[Q_{i 2 m} \cos \alpha_{i 2 m}+\frac{\xi_{i 2 m} M_{i m}}{D_{i b}} \sin \alpha_{i 2 m}\right] \cos \varphi_{i m} \\
\sum_{n=1}^{Z_{o b}}\left[Q_{o 1 n} \sin \alpha_{o 1 n}-\frac{\xi_{o 1 n} M_{o n}}{D_{o b}} \cos \alpha_{o 1 n}\right]= \\
\sum_{m=1}^{Z_{i b}}\left[Q_{i 2 m} \sin \alpha_{i 2 m}-\frac{\xi_{i 2 m} M_{i m}}{D_{i b}} \cos \alpha_{i 2 m}\right] \\
F_{i m}=\frac{1}{12} \pi \rho_{b} D_{i b}{ }^{3} D_{i p} \omega_{i p}{ }^{2} \\
F_{o n}=\frac{1}{12} \pi \rho_{b} D_{o b}{ }^{3} D_{o p} \omega_{o p}{ }^{2}
\end{gathered}
$$

Where, $D_{i p}, D_{o p}$ is the pitch diameter of double-decker bearing; $n_{i}$ is the speed of inner ring; $n_{m}$ is the speed of middle ring; $Q_{o 1 n}, Q_{o 2 n}, Q_{i 1 m}, Q_{i 2 m}$ is the contact force; $F_{i m}, F_{o n}$ is the centrifugal force; $\rho_{b}$ is the density of the ball; $D_{i b}, D_{o b}$ is the diameter of double-decker bearing; $\omega_{i p}, \omega_{o p}$ is the angular velocity; $\xi$ is the coefficient guide.

\section{Modified to the quasi statics model for double-decker bearing}

Taking the centrifugal expansion into consideration, the modified quasi-static model is established. The difference lies in the change of the deformation coordination relationship, and the formula is shown in (18)-(19). The Newton-Raphson method is used to solve nonlinear equations in the soft of Matlab.

$$
\begin{aligned}
& B_{i 1 m}=X_{i} \cos \alpha_{i 0}+\left(\lambda_{o r}-\Delta_{o r}\right) \cos \varphi_{i m}+\psi_{R 1} \\
& B_{o 1 n}=X_{o} \cos \alpha_{o 0}+\left(\lambda_{o r}-\Delta_{o r}\right) \cos \varphi_{o n}+\psi_{R 2}
\end{aligned}
$$

\section{Results and discussion}

\subsection{Bearing type selection}

The double-decker ball bearing is designated as 71901C and $71905 \mathrm{C}$, and the specific parameters are shown in Table 1.
Table 1. The parameters of the double-decker bearing $\square$

\begin{tabular}{|c|c|c|}
\hline \multirow{2}{*}{ Parameter } & \multicolumn{2}{|c|}{ Value } \\
\cline { 2 - 3 } & $\mathbf{7 1 9 0 1 C}$ & $\mathbf{7 1 9 0 5 C}$ \\
\hline $\begin{array}{c}\text { Diameter of ball } \\
\text { (mm) }\end{array}$ & 3 & 4.25 \\
\hline $\begin{array}{c}\text { Initial contact } \\
\text { angle ( }{ }^{\circ} \text { ) }\end{array}$ & 15 & 15 \\
\hline Number of ball & 17 & 21 \\
\hline $\begin{array}{c}\text { Groove curvature } \\
\text { coefficients of } \\
\text { inner raceway }\end{array}$ & 0.515 & 0.525 \\
\hline $\begin{array}{c}\text { Groove curvature } \\
\text { coefficients of } \\
\text { outer raceway }\end{array}$ & 0.515 & 0.525 \\
\hline $\begin{array}{c}\text { pitch diameter } \\
\text { (mm) }\end{array}$ & 18 & 33.5 \\
\hline
\end{tabular}

\subsection{Results analysis and comparison}

The Fig.3 and Fig.4 are shown the comparison of the change of the inner and outer contact angle in the inner bearing of double-decker bearing, as can be seen from the figures, the contact angle of considering centrifugal expansion is decreased compared to the original model. With the increase of rotational speed, the expansion of the centrifugal change gradually increase, this is because, with the increase of rotational speed, the centrifugal force of a ball is increased, the inner ring produced the radial deformation due to internal stress redistribution, which can lead to deformation of bearing internal coordination changed, and affect the dynamic performance of a bearing. With the increase of rotation speed, the amplitude of variation is larger. The influence of centrifugal expansion on the outer contact angle of inner bearing is greater than that of the inner contact angle. This is because the structural characteristics of the double-decker bearing, which has the function of the middle ring, the dynamic characteristics of the outer bearing will also make the contact angle change.

The Fig. 5 and Fig. 6 is the comparison of the change of the inner and outer contact angle in the outer bearing of double-decker bearing, as the figure shows, with the increase of rotational speed, the contact angle variation and variation range of the outer bearing are small, due to the speed distribution relationship between the inner and outer bearing, the influence of centrifugal force the influence of the external layer of ball bearing contact angle is less than the impact on the inner ball contact angle, so as the change of rotation speed, the outer ball contact angle variation, and variation range are smaller. The inner and outer contact angle of the outer bearing decrease with the increase of rotating speed after considering the centrifugal expansion rate, but the range of change is not obvious. This is because the working speed of the double-decker ball bearing is shared by the inner and outer bearings, and with the speed increased the contact angle changes less than that of the singlelayer ball bearing. 

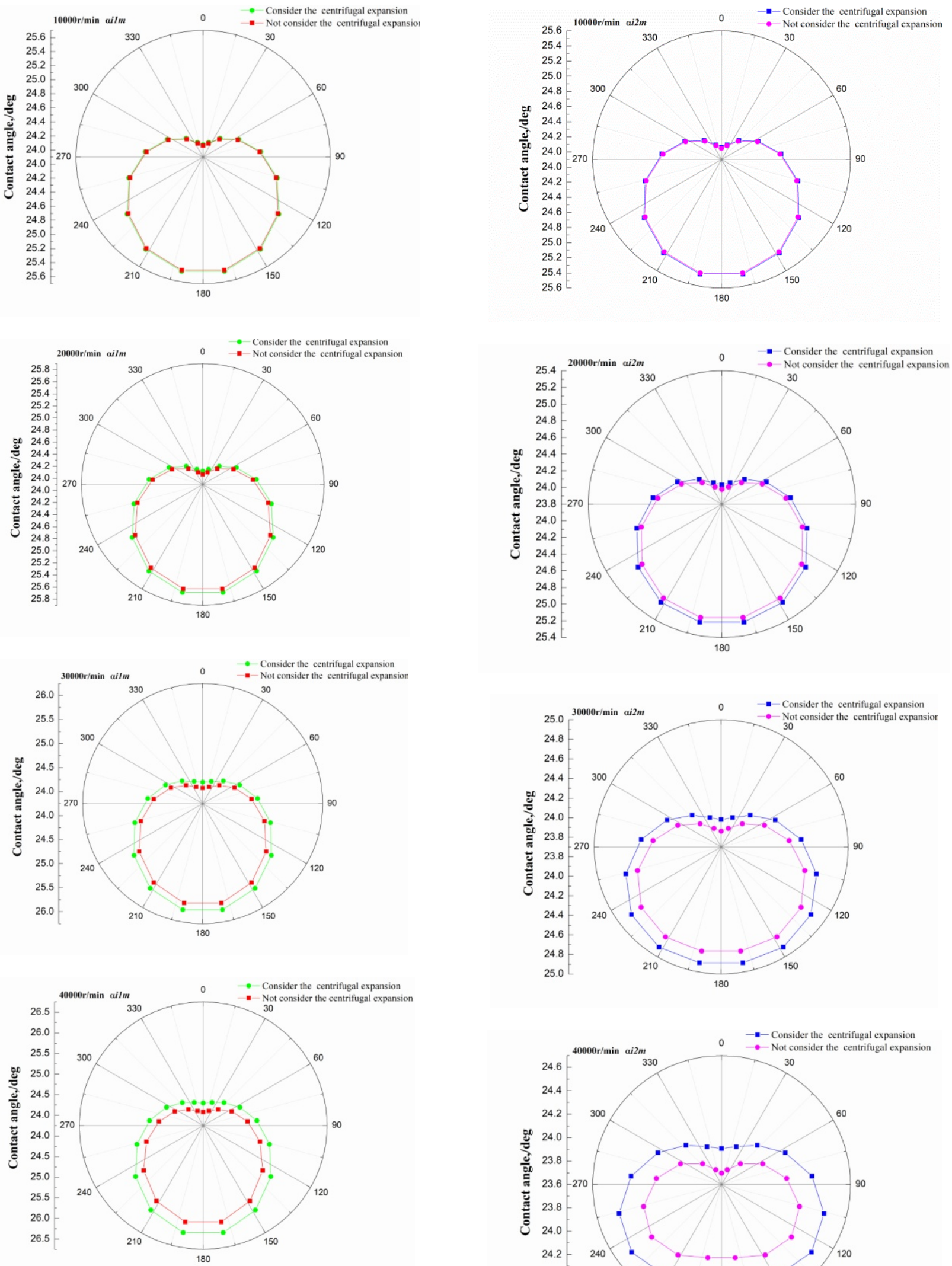

Fig. 3. The comparison of the change of the inner contact angle in the inner bearing of the double-decker bearing

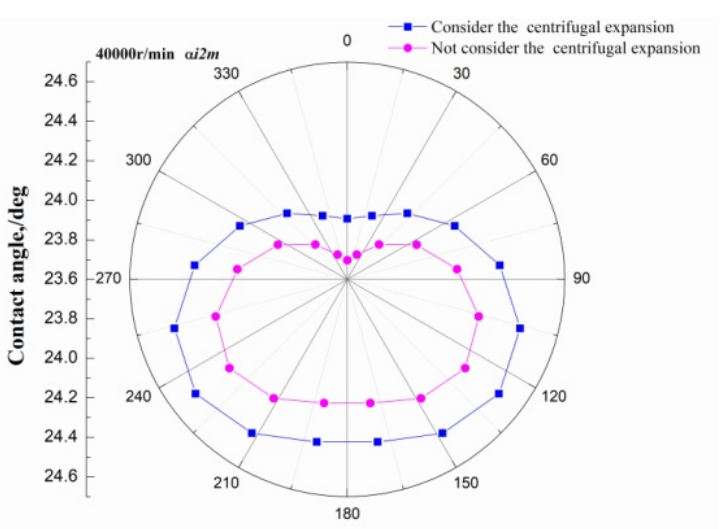

Fig. 4. The comparison of the change of the outer contact angle in the inner bearing of the double-decker bearing 

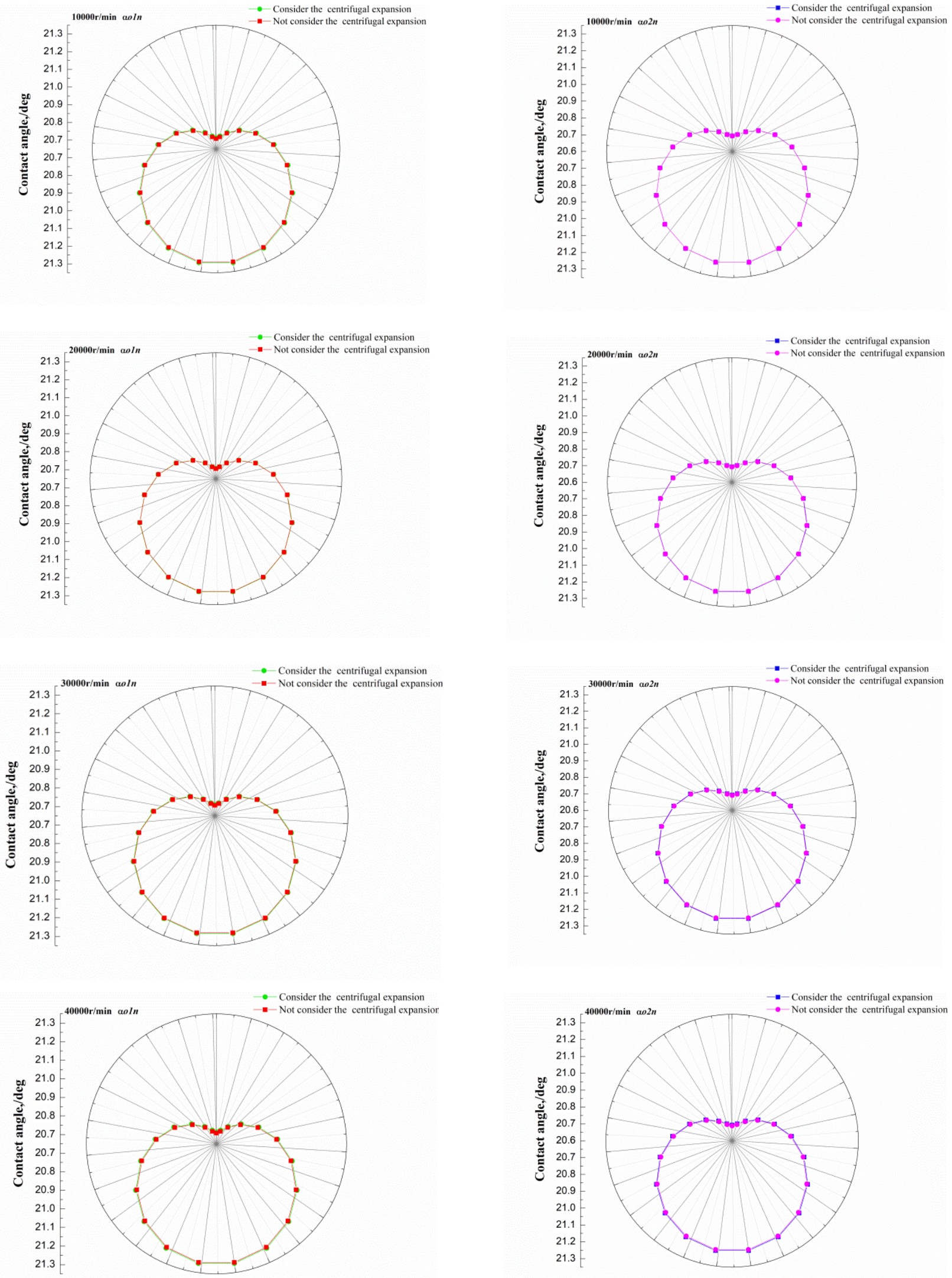

Fig. 5. The comparison of the change of the inner contact angle in the outer bearing of the double-decker bearing

Fig. 6. The comparison of the change of the outer contact angle in the outer bearing of the double-decker bearing

Fig. 7 shows the influence of the centrifugal inflation docking tentacles variation in the inner ring and ring under the different materials and the same speed. As is 
known from the figure, the contact angle considering centrifugal expansion is smaller than that without considering centrifugal expansion, when the inner ring and the middle ring are made of ceramic material, the change of the contact angle is bigger than the steel material, the biggest changes occur inner bearing external contact angle, which is decreased by $3.17 \%$.
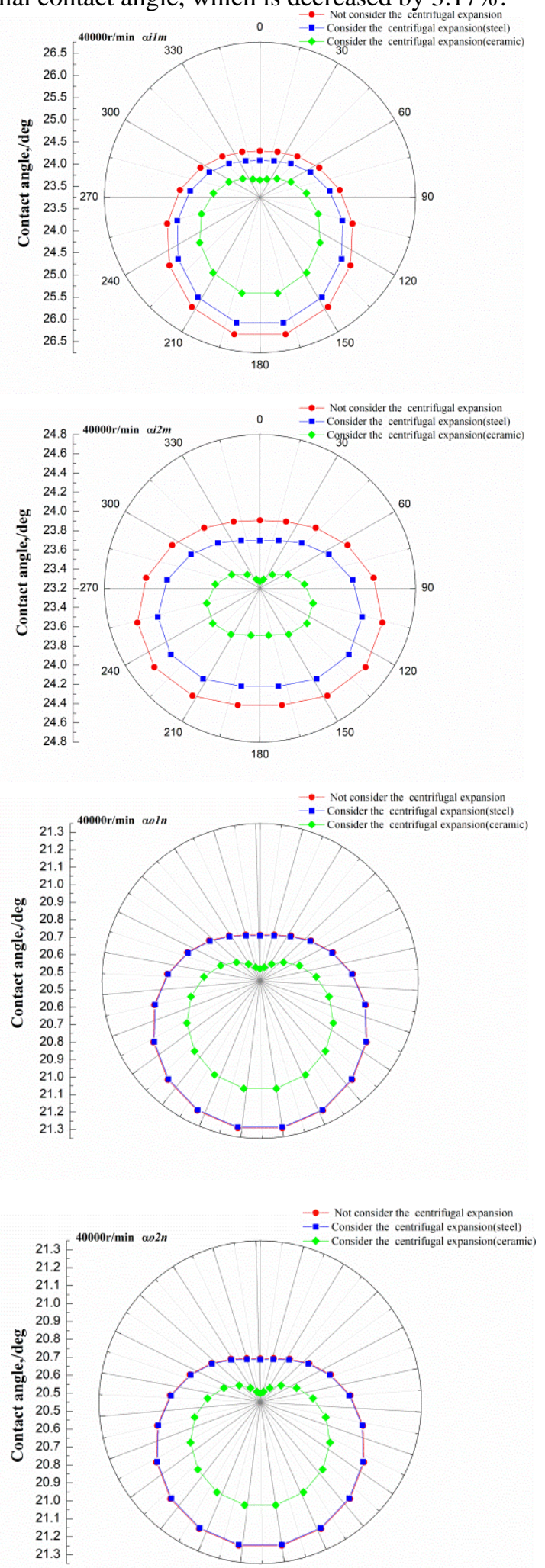

Fig. 7. The comparison of contact angle changes under different materials

\section{Conclusion}

In this paper, the double-decker bearing is taken as the research object, and the quasi-static model with the centrifugal expansion is modified. The analysis results are as follows:

(1) In the case of centrifugal expansion, with the increase of rotating speed, the contact angle of the inner and outer layers of the double-decker bearing decrease.

(2) In the case of centrifugal expansion, the influence on the contact angle of inner bearing is greater than that of outer bearing.

(3) With the increase of rotational speed, the influence of centrifugal expansion on the contact angle of doubledecker ball bearing gradually increases.

(4)Centrifugal expansion related to material parameters, select the appropriate materials can reduce the influence of centrifugal expansion on the dynamic characteristic, and the inner ring and ring choose ceramic materials caused amplitude of variation of the contact angle is bigger than that of the steel material, the biggest changes occur on the inner bearing external contact angle, which is decreased by $3.17 \%$, the high speed working conditions, centrifugal expansion effect on the dynamic performance of double-decker bearings should not be neglected.

\section{Acknowledgements}

This research is supported by the Jilin Province Science and Te chnology Development Program under Contract No.201702040 08GX.

\section{Reference}

1. Prashad, Har. 2001. A New Generation Double Decker High Precision Rolling Element BearingConcept, Development and Investigations. Tribology Transactions,44(2):203-208.DOI= http://10.1080/10402000108982449

2. Prashad, and Har.2004. "A Theoretical Approach to Evaluating the Performance Characteristics of Double-Decker High-Precision Bearings." Tribotest 10.3:251-263. DOI= http://10.1002/tt.3020100305 .

3. Prashad, and H.2006. "Solving Tribology Problems in Rotating Machines || A new generation of rollingelement bearing with an outline of its performance advantages." Solving Tribology Problems in Rotating Machines:228-242. DOI= http://10.1533/9781845691110.228.

4. Yu, Chengtao , L. Xu , and X. Yu .2014. "Research on the Mechanical Properties of "Z" Type DoubleDecker Ball Bearings." Journal of Tribology .DOI= http://136.1:111021-111028. 10.1115/1.4024844.

5. Yili Zhu, Yongchun Zhang, Chaowu Jin.2016.Research on the Mechanical Properties of a New "I” Type Double-Decker Ball Bearing. Journal of Tribology. DOI= http://10.1115/1.4031583. 
6. Li, Hongqi , and Y. C. Shin .2004. "Integrated Dyna mic ThermoMechanical Modeling of High Speed Sp indles, Part 1: Model Development." Journal of Man ufacturing Science \& Engineering .126.1:148-158. DOI= http://10.1115/1.1644545.

7. Cao Hongrui.2012.Centrifugal Expansion of Highspeed Spindle and Its Influences on Bearing Dynamic Characteristics. Journal of mechanical engineering. Vol.48 No.19. DOI= http://10.3901/JME.2012.19.059.
8. Zivkovic, Aleksandar , et al. 2015."Mathematical modeling and experimental testing of high-speed spindle behavior." International Journal of Advanced Manufacturing Technology 77.5-8:10711086. DOI= http://10.1007/s00170-014-6519-7.

9. Zhu Yi-li, Xu Long-xiang.2011. "The Radial Stiffness and Application of Double-Decker Ball Bearing." Key Engineering Materials. Vol 450,pp 353-356.

$\mathrm{DOI}=$ http://10.4028/www.scientific.net/KEM.450.3 